Objective of this work was to identify new biomarkers and establish serum protein fingerprint models for early diagnosis and preoperative evaluation of luminal stenosis severity of $\mathrm{CAD}$

Methods 48 eligible case subjects, having $>50 \%$ luminal stenosis in at least one major coronary artery (defined as coronary artery disease (CAD) by angiography), 39 patients with luminal stenosis between $25 \%$ and $50 \%$ (defined as coronary atherosclerosis), and 52 eligible health individuals, were recruited randomly in this study. The above 139 samples were analysed by Surface-enhanced laser desorption/ ionization time-of-flight mass spectrometry (SELDI-TOF-MS).

Results The clinical and biochemical characteristics in three groups matched well. To detect $\mathrm{CAD}$, thirty samples from patients with $\mathrm{CAD}$ (stenosis $\geq 50 \%$ ) and thirty-two samples from the control group were analysed and designated as pattern 1 . This pattern 1 model comprised ten potential biomarkers with $\mathrm{m} / \mathrm{z}$ of $4276,5326,4481$, $4320,4205,5814,5551,3689,5344$ and $6134 \mathrm{Da}$, respectively. The peaks at 5326, 5814, 5551, 5344, 6134 Da were expressed greatly in samples from patients with $\mathrm{CAD}$, but weakly expressed in healthy individuals; and the other five peaks were expressed greatly in healthy individuals but weakly expressed in patients with CAD. Pattern 1 had a specificity of $78.1 \%$ and a sensitivity of $80.0 \%$, as evaluated by leaveone-out crossvalidation. The remaining 38 serum samples, as a blind test set, were analysed on the second day to test pattern 1 . The specificity and sensitivity of the blind test were 83.3 and $72.2 \%$, respectively. To distinguish different severities of coronary artery stenosis, twenty-seven samples from patients with coronary atherosclerosis (between $25 \%$ and $50 \%$ ) were analysed and compared with thirty $\mathrm{CAD}$ (stenosis $\geq 50 \%$ ) patient samples. Pattern 2 was established similarly to distinguish coronary atherosclerosis (stenosis between $25 \%$ and $50 \%$ ) from CAD. Pattern 2 comprised seven potential biomarkers with $\mathrm{m} / \mathrm{z}$ of $3280,3689,5326,4289,6135,3164$ and $6439 \mathrm{Da}$, respectively. While the peaks with $\mathrm{m} / \mathrm{z} 5326$ and 6135 $\mathrm{Da}$, were expressed more significantly in CAD samples than in coronary atherosclerosis (stenosis between $25 \%$ and $50 \%$ ), the other five biomarkers appeared to be expressed in the opposite manner. This model had a specificity of $92.6 \%$ and a sensitivity of $93.3 \%$, as evaluated by leave-one-out crossvalidation. Pattern 2 was tested blindly in another 30 serum samples on the second day. The specificity and sensitivity of the blind test were $75.0 \%$ and $77.8 \%$, respectively.

Conclusion Taken together, the SELDI-TOF-MS technique combined with bioinformatics approaches can not only facilitate the discovery of better biomarkers for $\mathrm{CAD}$ and its severity, but also provide a useful tool for molecular diagnosis.

\section{e0088 MOUSE MODEL CARRYING LMNAE82K MUTATION IN MYOCARDIUM DEVELOPS DILATED CARDIOMYOPATHY AND APOPTOSIS}

doi:10.1136/hrt.2010.208967.88

Lu Dan, Lian Hong, Shao Haitao, Quan Xiongzhi, Huang Lan, Zhang Lianfeng. Institute of Laboratory Animal Science Chinese Academy of Medical Science

Objective To analyse the effects of LMNA ${ }^{\mathrm{E} 82 \mathrm{~K}}$ mutation on the transgenic mice heart.

Methods The transgenic mice were created by microinjection. Pathological changes in the heart of transgenic mice were observed by analyses from histologic, transmission electron microscopic, echocardiographic and ECG measurements. The expression of atrial natriuretic peptide (ANP), B-type natriuretic peptide (BNP), Col3 $\alpha 1$, lamin A/C, emerin, fas, caspase- 3 , caspase- 8 , caspase- 9 and cytochrome $\mathrm{c}$ in the myocardium of transgenic mice were determined by Reverse transcription PCR, Western Blot or immunofluorescence. Apoptiotic cells were detected by In situ terminal dUTP nick endlabelling (TUNEL) analyses.

Results Two lines, indicating cardiac-specific over-expression of h-LMNA ${ }^{\mathrm{E} 82 \mathrm{~K}}$, were established amongst the transgenic mice. All the changes of the LMNA ${ }^{\mathrm{E} 82 \mathrm{~K}}$ transgenic mice heart displayed a similar pathological phenotype to dilated cardiomyopathy. To sum up, the LMNA $^{\mathrm{E} 8 \mathrm{~K}}$ hearts exhibit thin-walled, dilated left and right ventricles, and had increased heart to body weight ratios as compared to WT hearts. Interstitial fibrosis, slight disarray of myofibrils, swollen mitochondria and loss of cristae as well as the loss of nuclear envelope integrity were observed in the myocardium of the LMNA ${ }^{\mathrm{E} 2 \mathrm{~K}}$ transgenic mice. The expression of fetal gene, BNP, was elevated in the LMNA ${ }^{\mathrm{E} 82 \mathrm{~K}}$ transgenic mice. Apoptosis in myocytes of LMNA ${ }^{\mathrm{E} 82 \mathrm{~K}}$ transgenic mice was detectable by TUNEL assay, apoptosis-related molecular signalling, especially in the Fas pathway, were activated by using Western Blot and immunofluorescence analysis.

Conclusions $\mathrm{LMNA}^{\mathrm{E} 82 \mathrm{~K}}$ transgenic mice developed DCM similar to the clinical features of human laminopathies and the most noteworthy was the apoptosis mechanism found in this transgenic mice. It may be a regulatory pathway providing an attractive therapeutic for the treatment of cardiomyopathy.

\section{e0089 THE ROLE OF PRENATAL CHRONIC HYPOXIA ON MYOCARDIAL ISCHAEMIA REPERFUSION INJURY IN ADULT RABBITS OFFSPRING}

doi:10.1136/hrt.2010.208967.89

Huang Ziyang, Yang Jingyang, Wang Zhenhua. The Second Affiliated Hospital of Fujian Medical University

Objective To evaluate the role of prenatal chronic hypoxia on myocardial ischaemia /reperfusion injury in adult rabbits' offspring and explore the relevant mechanism.

Methods The pregnant New Zealand rabbits were divided randomly into normoxic $(n=8)$ and hypoxic $\left(12 \% \mathrm{O}_{2}\right.$ from days 10 to 28 of gestation, $n=8$ ) groups. One male offspring of each maternal rabbit was randomly selected to study. The offspring rabbits were subjected to heat stress $\left(42^{\circ} \mathrm{C}\right.$ for $\left.15 \mathrm{~min}\right)$ at 6 months of age. After $24 \mathrm{~h}$, left anterior descending branches were excised and subjected to ischaemia for $30 \mathrm{~min}$ and reperfusion for $120 \mathrm{~min}$. Cardiac histopathological observation was performed by light microscope and electron microscope. The expression of heat shock protein 70 $\left(\mathrm{HSP}_{70}\right)$ in myocardium was detected by immunohistochemistry. Myocardial enzyme activity, apoptotic index and caspase-3 activity in myocardium were examined as well.

Results Ischemia-reperfusion after heat stress pretreatment increased myocardial enzyme activity, apoptotic index and caspase- 3 activity in prenatal chronic hypoxia rabbits (4720.31 $\pm 744.39 \mathrm{IU} / \mathrm{l}$, $1849.13 \pm 416.58 \mathrm{IU} / 1,40.43 \pm 5.03 \%, 12.43 \pm 1.77$ unit, respectively)

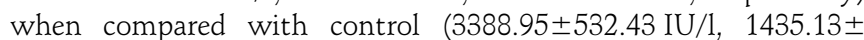
$92.08 \mathrm{IU} / 1,34.40 \pm 4.66 \%, 10.58 \pm 1.42$ unit, respectively). Heat stress pretreatment induced $\mathrm{HSP}_{70}$ significant expression in left ventricular myocardium was not observed in prenatal chronic hypoxia rabbits but in normoxic control rabbits.

Conclusions Prenatal chronic hypoxia inhibits $\mathrm{HSP}_{70}$ synthesis in the heart of adult offspring in response to body heat stress, which might insult cardioprotection against ischaemia-reperfusion injury.

\section{e0090 EFFECT OF GRANULOCYTEMACROPHAGE COLONY STIMULATING FACTOR ON COAGULATION AND FIBRINOLYSIS IN RABBIT MODEL WITH ILIAC DAMAGED BY BALLOON}

doi:10.1136/hrt.2010.208967.90

Ma Xiaojing, Zhang Xinghua. Provincial Hospital Affiliated To Shandong University

Objective Endothelial dysfunction after percutaneous coronary intervention (PCI) played a key role in perturbations of haemostatic equilibrium. Granulocyte-macrophage colony stimulating factor 